\title{
FUNDAMENTAL STUDIES FOR AUTOMATIC SCORING OF BRAIN STEM RESPONSE
}

\author{
SATOSHI KOLZLMI, M.D.
}

Department of Otolaryngology, Facality of Medicine, Shinshu University, Matsumoto.

(Director: T. Suzuki, M,D.)

A preliminery investigation was made for establishing an atomatic method of scoring of brain stem response. (BSR). Aperaged BSR to click stimuli was recorded in 7 normal young children and 4 normal adults. They were scored visually and by 3 different methods of amplitude messurement.

The stimuli were given to the subject through a loudspeaker at intensity levets of $10,30,30$ and $40 \mathrm{~dB}$ above mean subjective thresholds of normal adults (HL) for young children and of 0 , 10, 20 and $30 \mathrm{~dB}$ (HL) for adv subjects. Ten averaged responsea were recorded with each stimulus intensity and additional 10 traces were-made without stimulation. Thus 50 traces were obtained per subject.

The amplitudes of the responses of all traces were measured asing 3 diffezent nethods shown in Fig. 1 ( $A, B$. C). Then 5 percent rejection limits were calculated of the amplitudes of 10 control traces measured by the 3 methods. These values were used as amplitude criteria for judging the presence or absence of a response. Responses whase amplitude exceeded the above-mentioned criteria were scored positive, and the rate of positive responses in the 3 methods were compared with that in the visual acoring.

Regults obtaiaed were as follows:

1) In young child group, Method B and C achieved almast similar detectability of the response as did visual scoring, whereas the rate of positive responses in Method A was signifcantly lower than those in $\mathrm{B}$ and $\mathrm{C}$.

2) In adult groug, on the contrary, nu such a significant difference was found in the response detectability_among the 3 methods, though all of the metbods showed 15 to 40 percent lower detectability than that of visual scoring.

3) We thus concluded that Method B was regarded as the most useful procedure for measurise response amplitude automatically.

A79-0591. I3522

\section{聴性眇幹反応の自㖶判定に関する基礎的䚁察*}

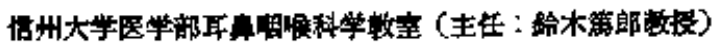

$$
\text { 小泉揰 }
$$

it

1970年, Sohmer \& Feinmesser ${ }^{21}$ 拈よ $\psi^{*}$ Jewett
第100禁

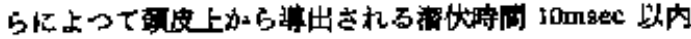

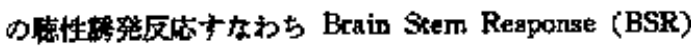

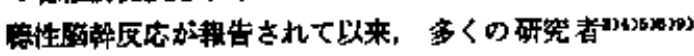

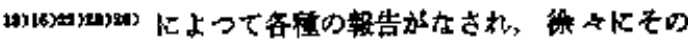




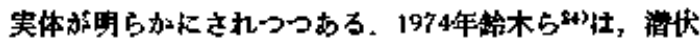

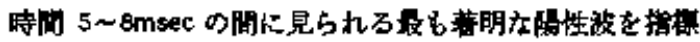

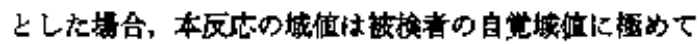

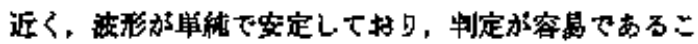

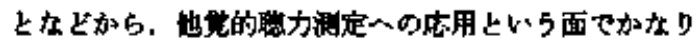

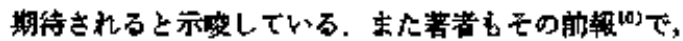

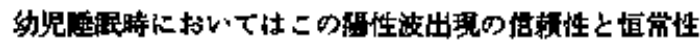

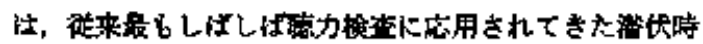
閶 $50 \mathrm{msec}$ 以上のいわける Slow Vertex Potential に

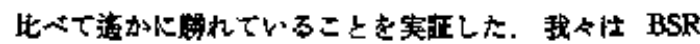

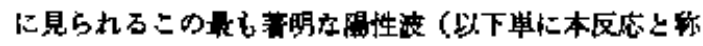

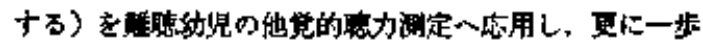

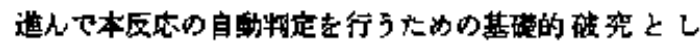

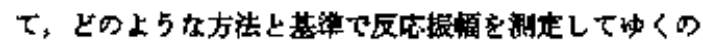

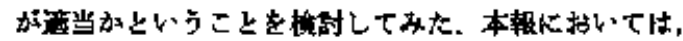
加策反応の加の所定の 2 点同の后位差がある一定值を

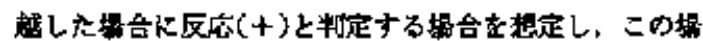

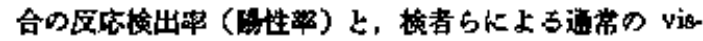

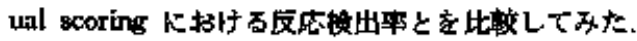

方 法

1、设镍者

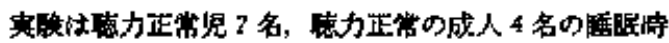
という策件下に行かつた．成人住自然睡眠であるが，幼

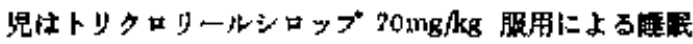

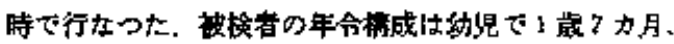

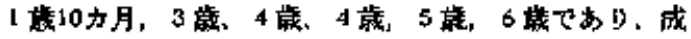

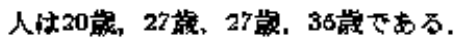

2. 加算被形心作成

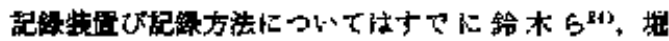

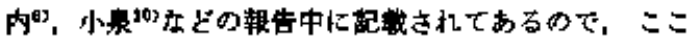

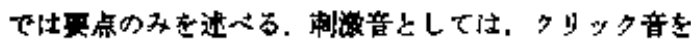

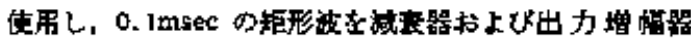

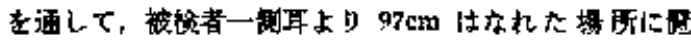

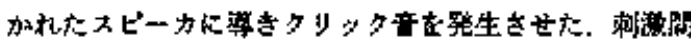
两は 50msec，出力は原則上して正常等力成人数名の平

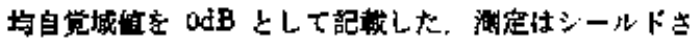
れた防音室を行なわれた。

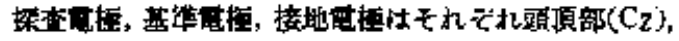

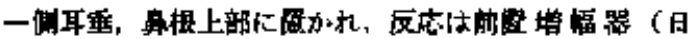

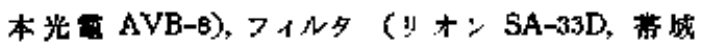
1,8〜2800H2,30dB 畦愊)を们し、コンビュータ（日本

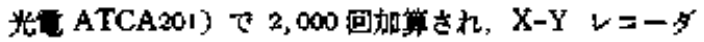

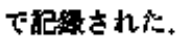

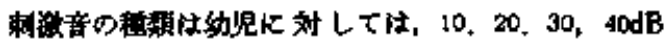

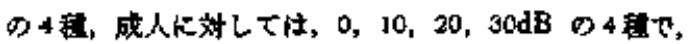

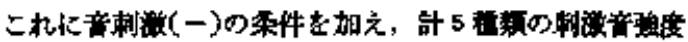

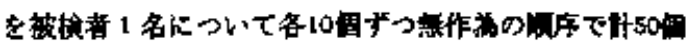
のサンプル妾作成した.

3、提湿の激定

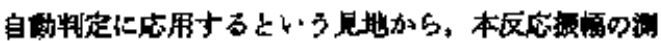

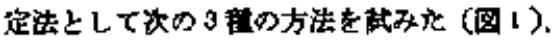

因 1 报腰の调定方法 A， B，C

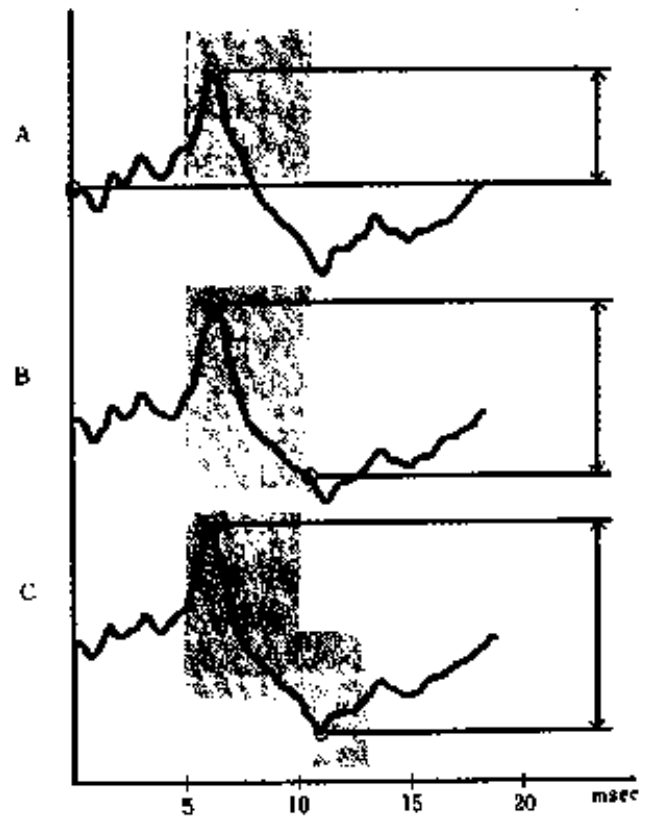

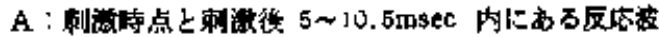

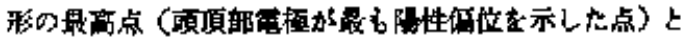

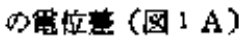

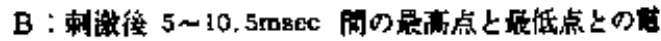
位羛 (园1B)

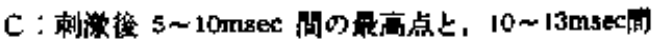

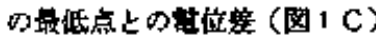

4. 反花判定の基䇤

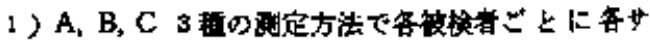

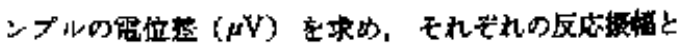
LA.

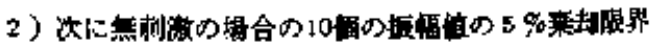

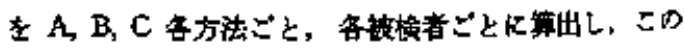

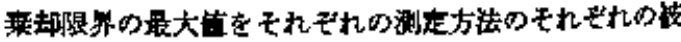
榙者の反灾の有率の critical value とした. 


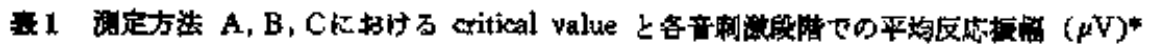

\begin{tabular}{|c|c|c|c|c|c|c|c|c|}
\hline & & critical value & NS** & $0 \mathrm{~dB}$ & $10 \mathrm{~dB}$ & $20 \mathrm{~dB}$ & $30 \mathrm{~dB}$ & $40 \mathrm{~dB}$ \\
\hline \multirow{3}{*}{$\begin{array}{l}\text { 肾 } \\
\text { ( } 7 \text { 明) }\end{array}$} & $\mathbf{A}$ & 0.337 & 0.105 & - & 0.145 & 0.214 & 0.288 & $0 . \$ 43$ \\
\hline & $\mathrm{B}$ & 0.240 & 0.136 & - & 0.210 & 0,307 & 0.423 & 0.535 \\
\hline & $\mathrm{c}$ & 0.247 & 0.111 & - & 0.196 & 0.312 & 0.460 & 0,609 \\
\hline \multirow{3}{*}{ 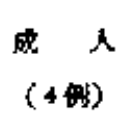 } & A & 0.338 & 0.094 & 0.124 & 0.383 & 0.638 & 0.923 & - \\
\hline & $\mathbf{B}$ & 0.326 & 0.362 & 0.219 & 0.381 & 0.477 & 0.600 & - \\
\hline & C & 0.372 & 0.140 & 0.231 & 0.432 & 0.580 & 0.771 & - \\
\hline
\end{tabular}

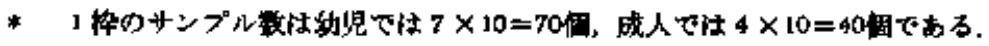

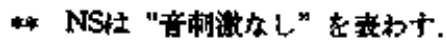

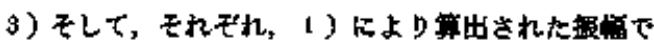

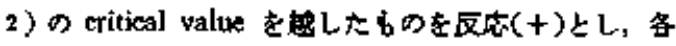

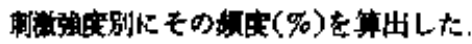

4) visual scortng it 3 人の桧者によつで行かわれ，

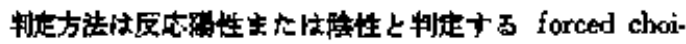
ce nethod 站用いた。

\section{店}

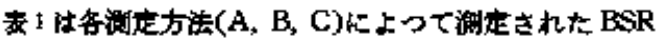

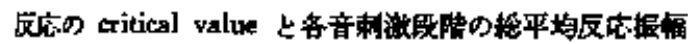
を示したらのである. 纤見に批いてけ critiol value は

B2 测定方法 A, B, C \& Visual Scoring: 门平均反度挨出虫

(就呪 7 人)
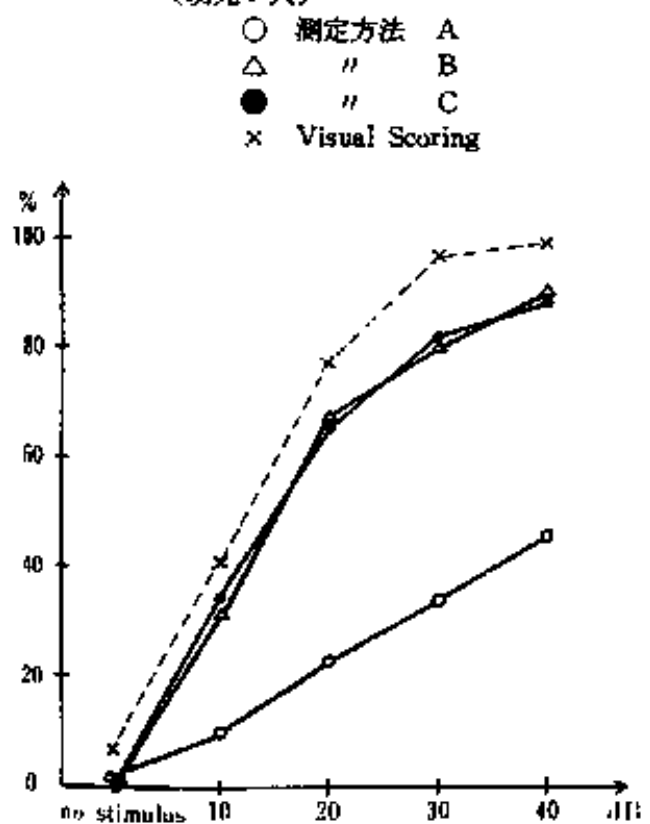

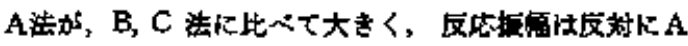
法の放が B，C法に比へて小さく斿っている. 一方成人

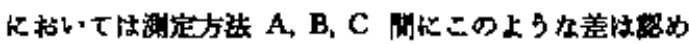
G九ていth.

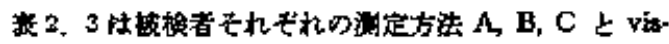

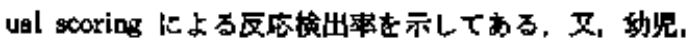

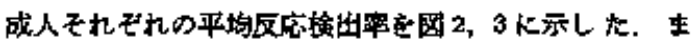

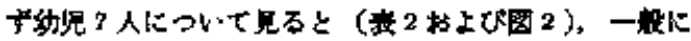

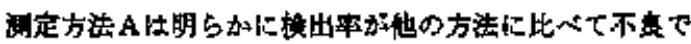

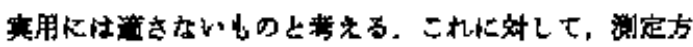

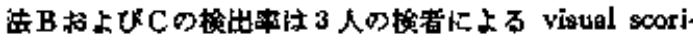

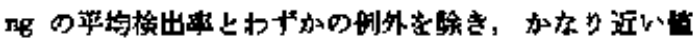
を示して括り，Z名の平姆直を見ても，洞定方洗 Bの

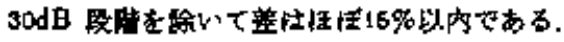

国 9 测定方法 A, B , C 上 Visual Scoring の平均反店识出

(成人 4 人)

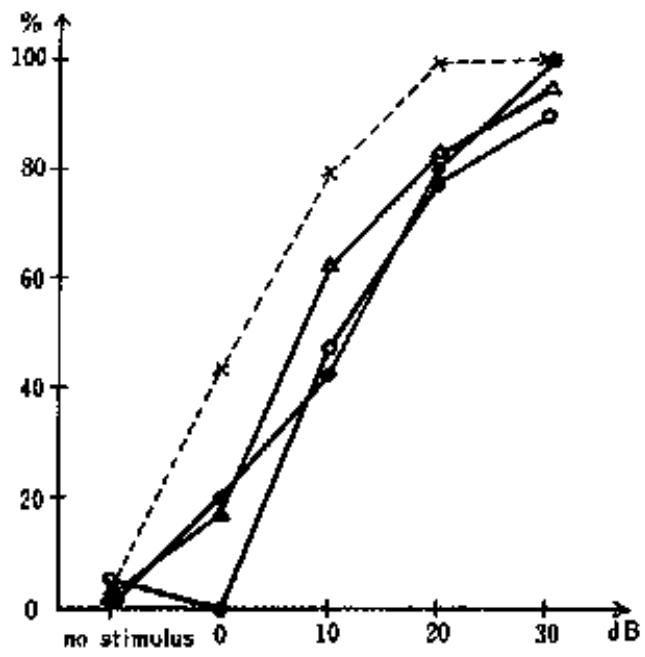




\begin{tabular}{|c|c|c|c|c|c|c|c|c|}
\hline \multirow{5}{*}{ 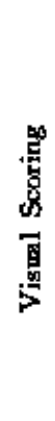 } & $\%$ & 88 & 8 & 8 & 8 & 8 & 8 & $\begin{array}{l}\text { s. } \\
\text { so } \\
0\end{array}$ \\
\hline & 8 & $\dot{\alpha}$ & 8 & 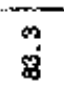 & 8 & 8 & 8 & के \\
\hline & $\&$ & $\begin{array}{ll}0 & 8 \\
7 & 8\end{array}$ & 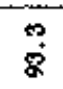 & 8 & $\begin{array}{l}m \\
\dot{s}\end{array}$ & 8 & $\mathscr{8}$ & $\frac{1}{8}$ \\
\hline & 0 & 운 8 & 8 & $\ddot{\xi}$ & $\ddot{m}$ & vin & $\vec{d}$ & 采 \\
\hline & n & 00 & 0 & 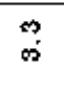 & $\overrightarrow{0}$ & $\vec{b}$ & $\ddot{\sigma}$ & S \\
\hline \multirow{5}{*}{0} & 옴 & 88 & 8 & 字 & 8 & 8 & 8 & 㖞 \\
\hline & 8 & 呂 8 & 8 & 0 & 8 & 8 & $g$ & $\frac{7}{7}$ \\
\hline & 8 & ㅇ 8 & $\$$ & 0 & 8 & $\$$ & 8 & $\overline{8}$ \\
\hline & 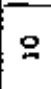 & 98 & 요 & 0 & 유 & 8 & g & 等 \\
\hline & 公 & $\circ \quad 0$ & 0 & 0 & 0 & 0 & 0 & 0 \\
\hline \multirow{5}{*}{$\boldsymbol{\infty}$} & 웅 & 용 & 苞 & 8 & $\cong$ & 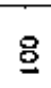 & 品 & $\begin{array}{l}8 \\
8 \\
8\end{array}$ \\
\hline & 8 & 88 & 8 & 8 & 8 & 8 & 怘 & $\begin{array}{l}8 \\
\dot{8}\end{array}$ \\
\hline & ले & $\circ \quad \&$ & 8 & $q$ & 8 & 8 & 욤 & 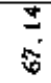 \\
\hline & D & 9 움 & 8 & 只 & g & ํㅗ & 9 & 穴 \\
\hline & $\begin{array}{l}n \\
z\end{array}$ & 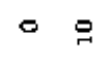 & 0 & 0 & $a$ & 0 & 0 & $\stackrel{9}{7}$ \\
\hline \multirow{5}{*}{$<$} & 웅 & $\circ \quad 8$ & 8 & 0 & $\therefore$ & 8 & สี & $\vec{q}$ \\
\hline & 8 & $\circ \%$ & 8 & 0 & 8 & 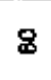 & 0 & $\begin{array}{l}\text { ì } \\
\text { j. }\end{array}$ \\
\hline & 8 & $\circ$ \& & 8 & 0 & है & 8 & 0 & $\begin{array}{l}\text { \$ } \\
\text { बें }\end{array}$ \\
\hline & ? & $0 \subseteq$ & 9 & 9 & 0 & 윰 & $\theta$ & $\begin{array}{l}8 \\
0\end{array}$ \\
\hline & n & $\circ 9$ & & & & & 0 & \% \\
\hline 躃 & 墨垔 & 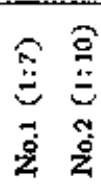 & $\begin{array}{l}8 \\
8 \\
0 \\
z\end{array}$ & $\begin{array}{l}\text { z } \\
0 \\
0 \\
\dot{z}\end{array}$ & 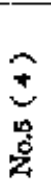 & $\begin{array}{l}6 \\
6 \\
6 \\
8 \\
2\end{array}$ & $\begin{array}{l}\hat{0} \\
0 \\
0\end{array}$ & 接 \\
\hline
\end{tabular}

\begin{tabular}{|c|c|c|c|c|c|}
\hline \multirow{5}{*}{ 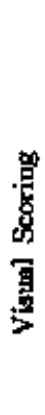 } & 8 & 88 & 8 & 8 & 8 \\
\hline & 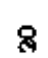 & $8 \stackrel{\circ}{\circ}$ & 8 & 8 & $a$ \\
\hline & 9 & \& 8 & 8 & 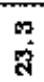 & 2 \\
\hline & 0 & 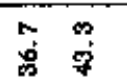 & : & ્ે & 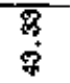 \\
\hline & 告 & it & 0 & 0 & 节 \\
\hline \multirow{5}{*}{0} & 呙 & $8 \&$ & 8 & 8 & 8 \\
\hline & $\mathscr{8}$ & 용 & 8 & 8 & $\begin{array}{l}8 \\
8\end{array}$ \\
\hline & 9 & 우 유 & $\mathscr{R}$ & 0 & 8 \\
\hline & 0 & 08 & 用 & 용 & $\begin{array}{l}8 \\
\dot{8}\end{array}$ \\
\hline & z & 90 & $\circ$ & 0 & $\stackrel{8}{R}$ \\
\hline \multirow{5}{*}{ 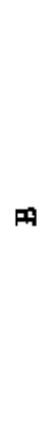 } & 8 & 89 & 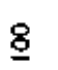 & 品 & $\begin{array}{l}8 \\
\text { 며 }\end{array}$ \\
\hline & व & 88 & 8 & 옥 & 8 \\
\hline & 9 & $8 \%$ & 8 & 0 & $\begin{array}{l}\text { 8 } \\
\text { तु่ }\end{array}$ \\
\hline & 0 & 09 & & 8 & $\begin{array}{l}6^{\circ} \\
\vdots \\
=\end{array}$ \\
\hline & n & 90 & 0 & 0 & 量 \\
\hline \multirow{5}{*}{4} & $g$ & $\$ 9$ & 8 & 8 & $\begin{array}{l}8 \\
8\end{array}$ \\
\hline & กิ & $8 \mathrm{R}$ & 8 & 유 & S \\
\hline & 9 & 89 & 8 & 0 & $\begin{array}{l}8 \\
5\end{array}$ \\
\hline & 0 & 00 & 0 & 0 & 0 \\
\hline & z & 00 & & 0 & $n$ \\
\hline 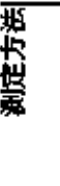 & 倠 & 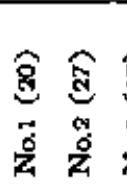 & 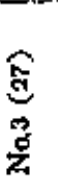 & 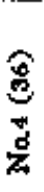 & $\begin{array}{l}\text { S } \\
H\end{array}$ \\
\hline
\end{tabular}




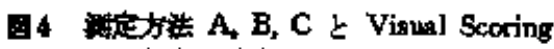
との判定一使庆

(奻児 7人)

O 方浩 A
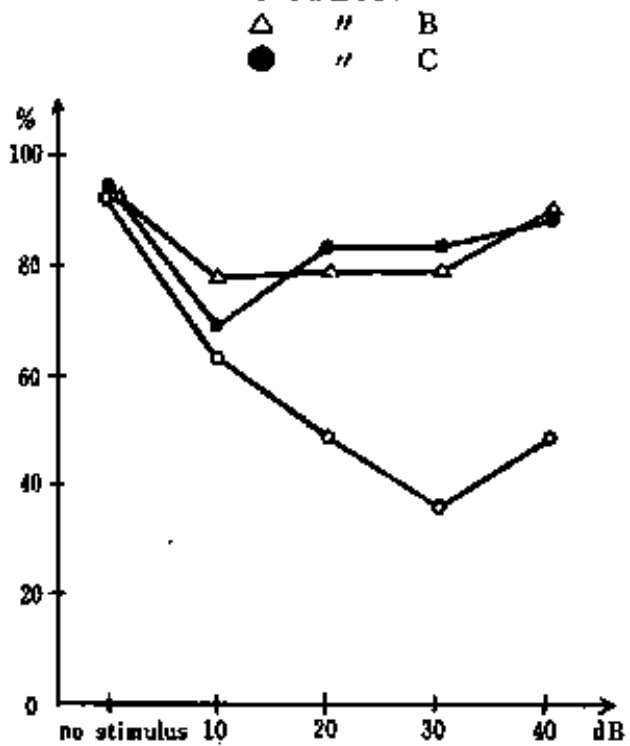

因5 湖走方 A, B, C と Visual Scoring 七の判定一政度

（成人 4 人)

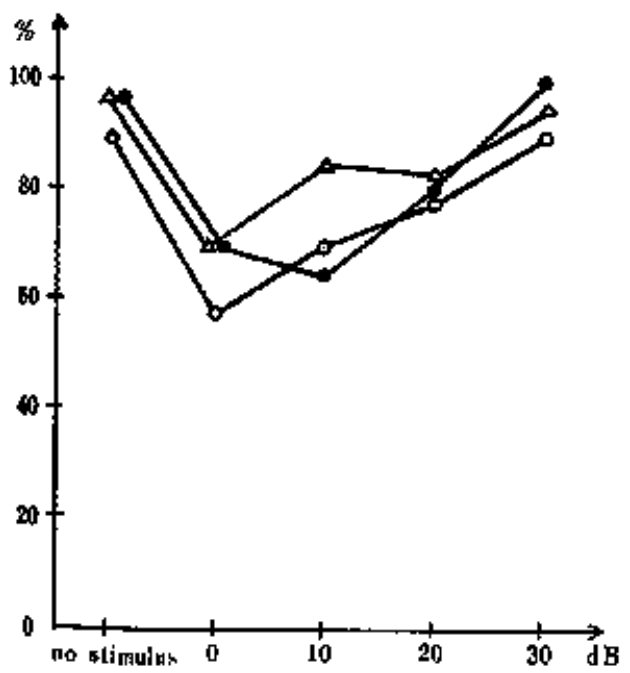

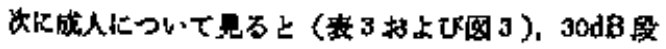

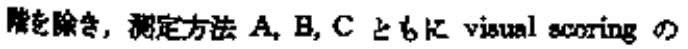

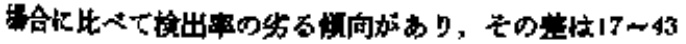

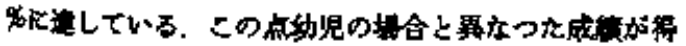

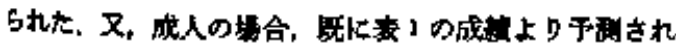

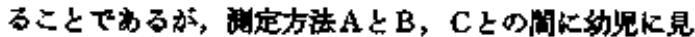

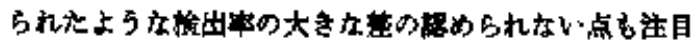
される。

炊に visual scoring と谢定方法 $\mathrm{A}, \mathrm{B}, \mathrm{C}$ たよる判定

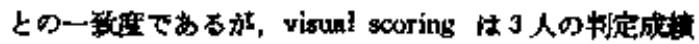

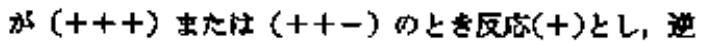

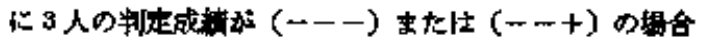

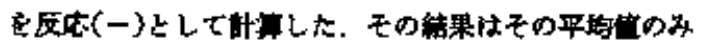

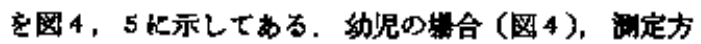

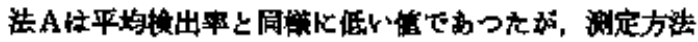

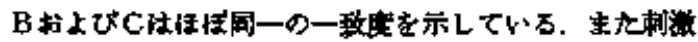

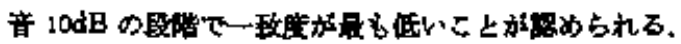

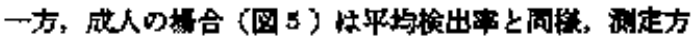

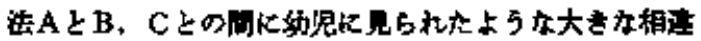
はなかった。.

\section{雭}

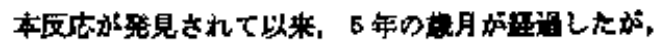

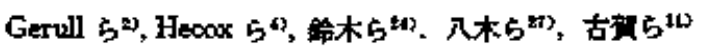

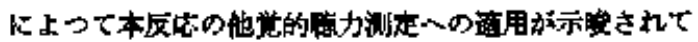

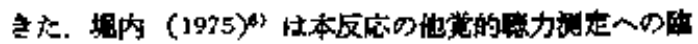

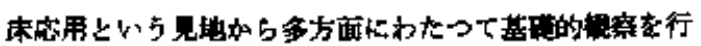

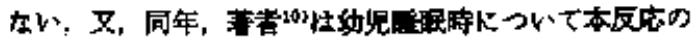

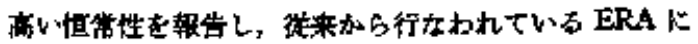

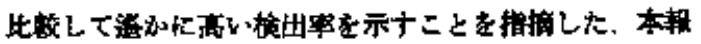

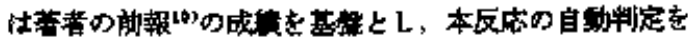

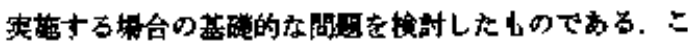

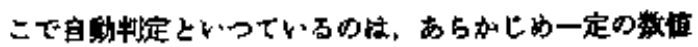

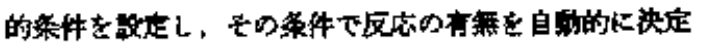

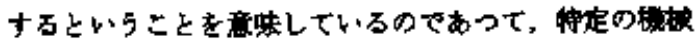
的据作吉定しているわりではない。

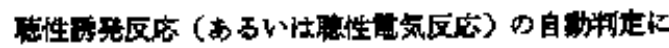

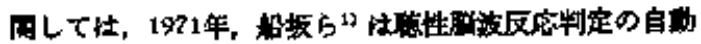
化に一、て速心，成人用 auto-EEG-audiometer O作

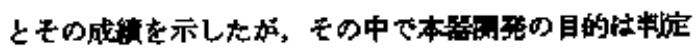

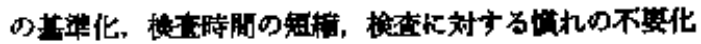
たとでると述へ， visual scoring と machine

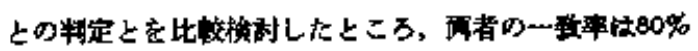
以上にる年り，十分宾用になるものとしている. 又,

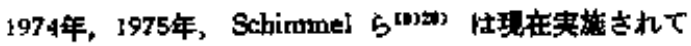

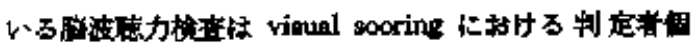

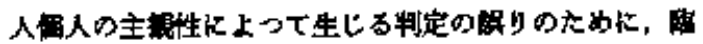

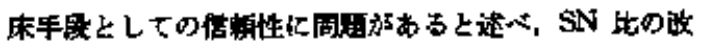

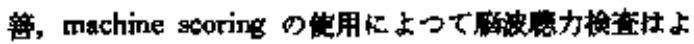




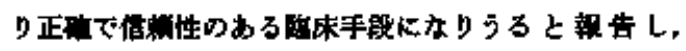

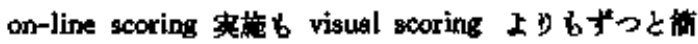

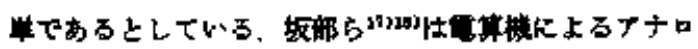

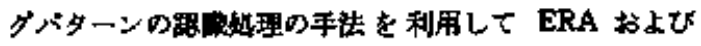
BSR の波形を起理することを诗みている，束た， Leitner ${ }^{(2)}$ it Wehrmann 0 stachastisch-ergodische Konv-

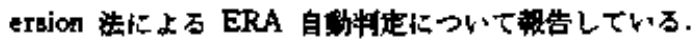

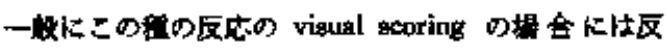

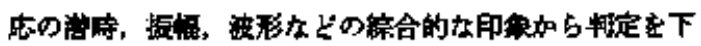

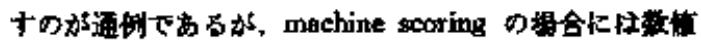

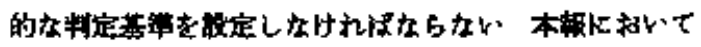

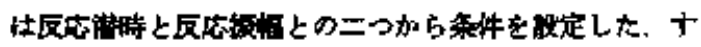

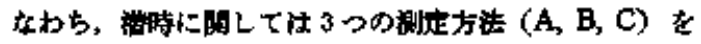

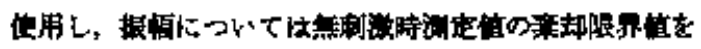
critical value として仿用してみた.

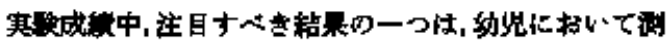
定方法Aによる模出事㘦模しく不良だつたことである。

沙定方法A

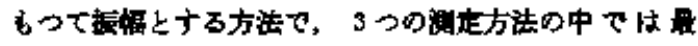

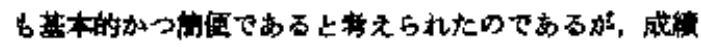

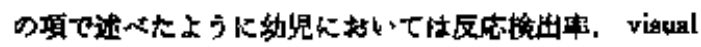

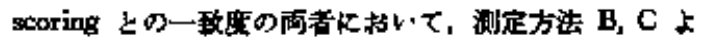

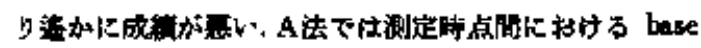

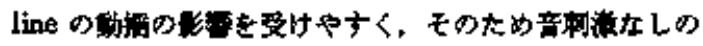

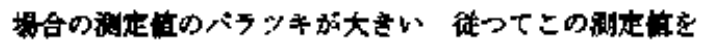

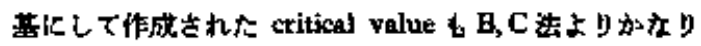

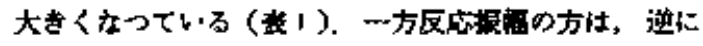

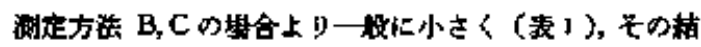

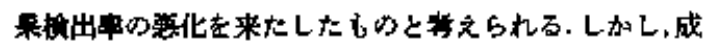

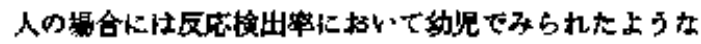

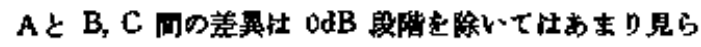

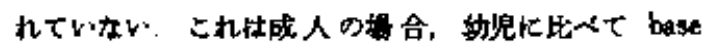

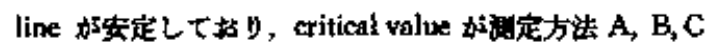

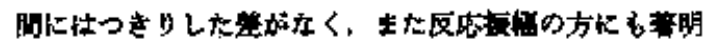

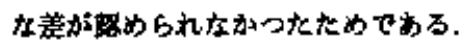

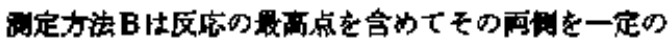

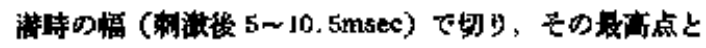

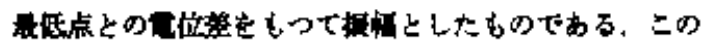

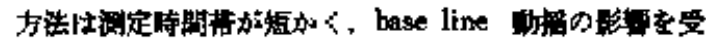

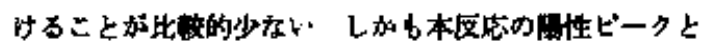

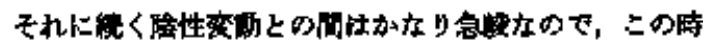

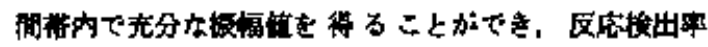

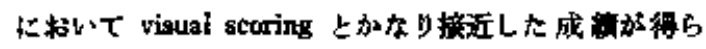

れたるのと思か机る。 しかし成人で仗 visual scoring $\mathrm{K}$

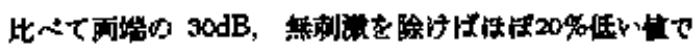

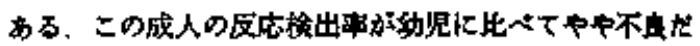

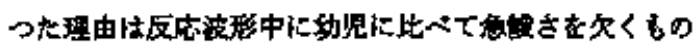

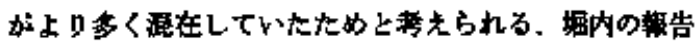

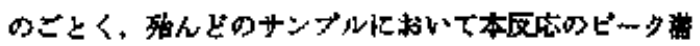

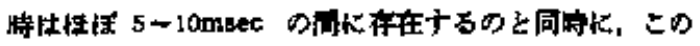

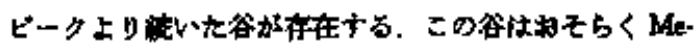

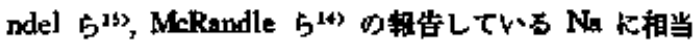

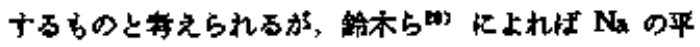

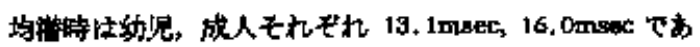
D，纤坚の方が成人に比へて短いこと加指商吉れ下い

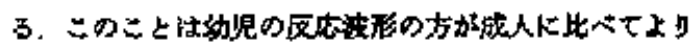
祭皮なカーフを描いていることを示している.

著橥位以前上り BSR-Na componeats (本反店の最高

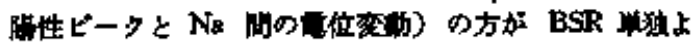

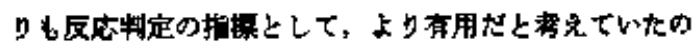

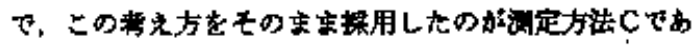

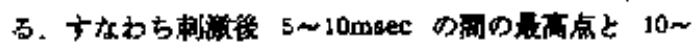

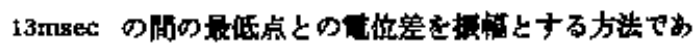

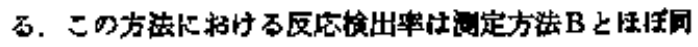

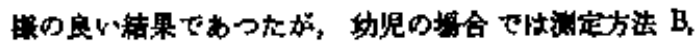

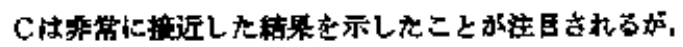

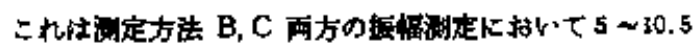

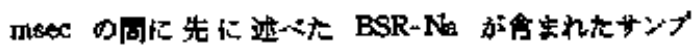

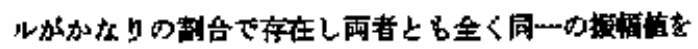

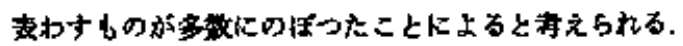

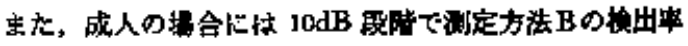
比デて 15 20\% 不良であつなが：このこと恃成人の

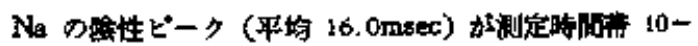

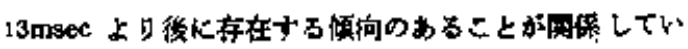
万ものと思われる。

visual scoring と期定方法 A，B，Cに上万物定との

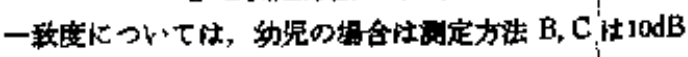

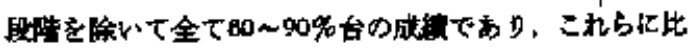

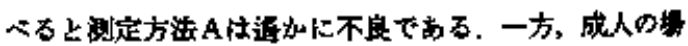

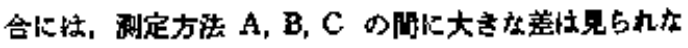

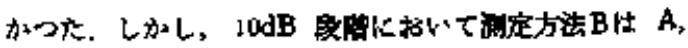

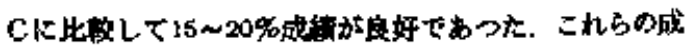

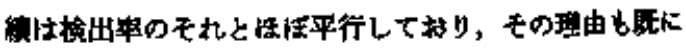
速バたことでつをている思う。

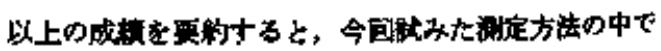

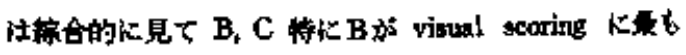




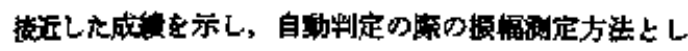

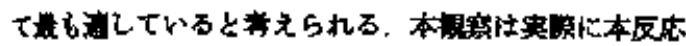

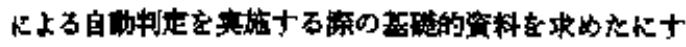

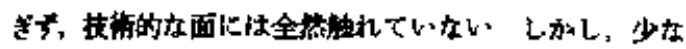

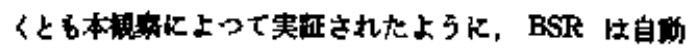

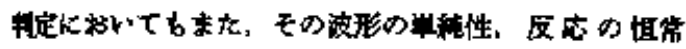

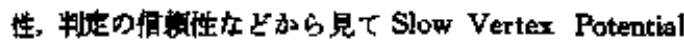

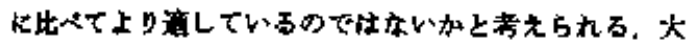

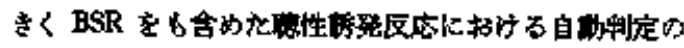

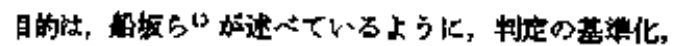

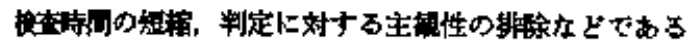

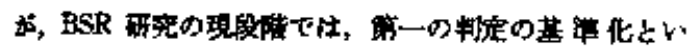

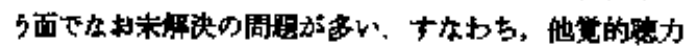

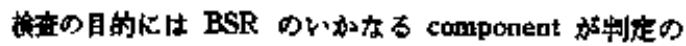

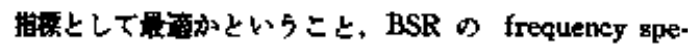

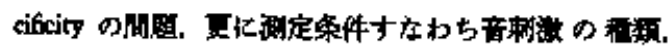

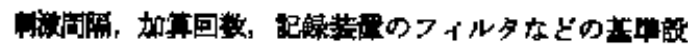

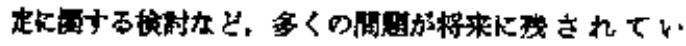

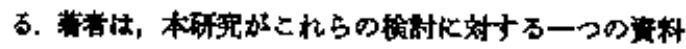
として敷っことがかれ涬いであると考える。

\section{船 8}

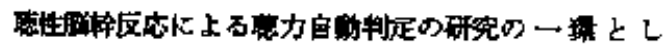

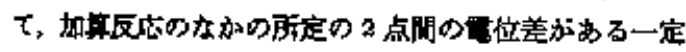

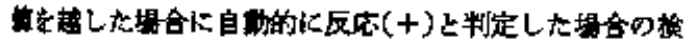

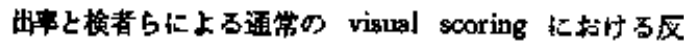

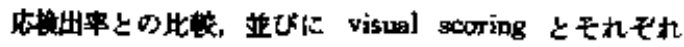

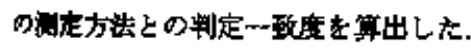

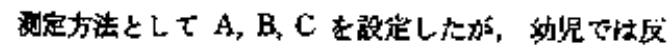

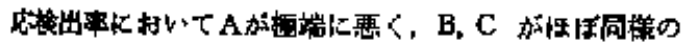

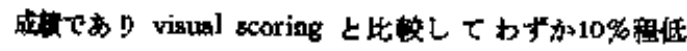

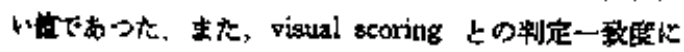

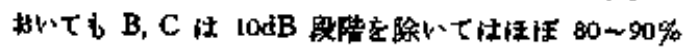

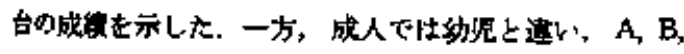

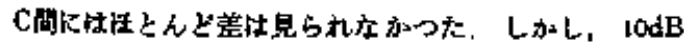

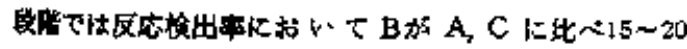

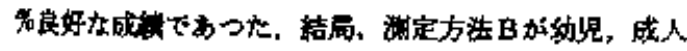

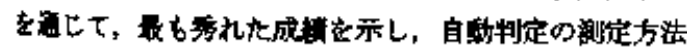
上しては㩆であると若えられた。

\section{女 到}

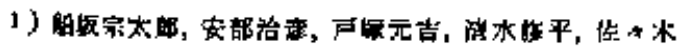

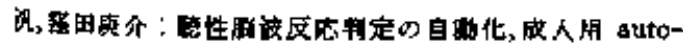

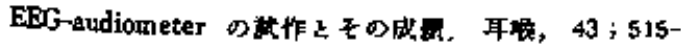
520, 1921 .
2) Gerull, G., Giesen, M., Mrowoinski, D.u, Rutdolph, $N$ : Untersuchung eines frthen, von der kopflaut ableitberen Potentials far die objektive Audiometrie. HNO, $20 ; 339-343,1972$.

3) Gerull, G., Giesen, M., Mrowinski, D.u. Rudolph, N.: Diagnostily von Hỡstörungen durch ERA mit sebr frühen Potentialen. HNO, 22 ; 230-225, 1974. 4) Hecrx, K. and Galambas, R.: Brain stem auditory evolked responseg in human infantg and adultk, Arch. Otolaryng, $99: 30-38,1974$.

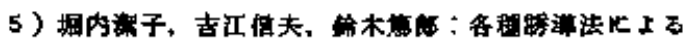

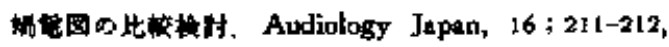
1973.

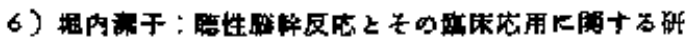

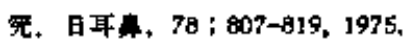

7) Jewert, D.L., Romano, M.N. and Williston, J.S.: Human auditory evoked pocentials: Possible brin atem components detected on the scalp. Science. N.Y., $167 ; 1517-1518,1970$.

s) Jeweth, D.L.: Volune-cosducted potentials in respense to auditory stimuli as detected by avernging in the cat. Electroenceph. Clin. Neurophyziol., 20 ; 609-618, 1970.

9) Jewett, D.L. and and Williston, J.S.: Auditoryevclked for fields averaged from the stalp of human. Brain, 94; 581-596, 1971.

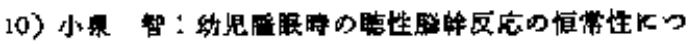

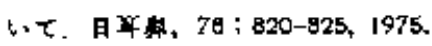

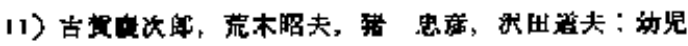

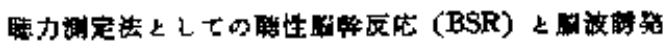

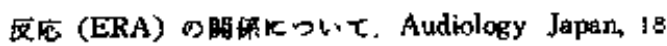
; 310-326, 1975 .

i2) Leitner, H. Eìn neues Verfabren zur automatis. chen Auswertung der ERA mit Hilfe der stochastisch-ergndischen Konversion (SEC). Largng. Rhinod., 54 ; 677-681, 1975.

13) Lvv, A. and Sohmer, H.: Sources of averaged neural responses recocdied in animal and human sabjects during cochlear audiometry (electro-cochl eogram). Arch Klin. exp. Ohr.-, Nas- u. Kehlk. Hejlk., $210 ; 79-90,1972$.

14) McRandle, C.C. Smith, M.A., and Goldstein, R.:

Early averaged electri-encephalic responses to clic. 
ls in neonatex, Ann. Otol, 69: 695-702, 1974.

15) Mendel, M.I. and Goldsteiti, R.: Stability of the early components of the averaged electroencephalic reopouse. J. Speech Res., 12 ; 351-361, 4969.

16) Pictor, T.W. Hillyard, S.A., Krawss, H.L. and Gatambos, $R$ : Human atxditory evoked potentiala. I: Evaluation of components. Electroenceph. clip. Neurophysiol., $36 ; 179-190,1974$.

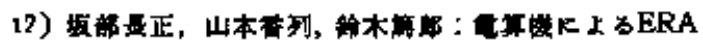

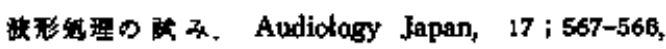
1974 .

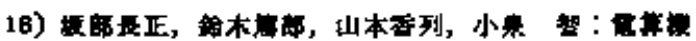

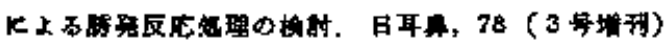
; 67, 1975.

19) Schimal, H, Ropin, I. and Cohen, M.: Improving evolted regponse ardiometry with special reference to the we of machine scaring. Audiolnay, 13 ; 33-65, 1974.

20) Schimet, H., Rapin, I. and Cahon, M.: Iroproving evoked response audiometry. Results of normative studies for mactione scoring. Audiology, 14 ; 466-479, 1975.

21) Sahmer, H, and Feimester, M.: Cochlear and $\omega$ rtical audiometry. Israel J. Med. Sci., 6 ; 219-223, 1970.

22) Sohwer, $H$, Feinuesser, M, Bamberger-Tell, L,
Lev, A. and David S.: Routine use of cacblear audiometry in infants with uncertain diagnosis. Ann Otol., 81 ; 72-75, 1972.

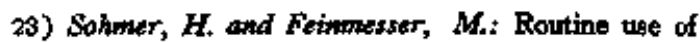
electrocochleography (cochlear audiometry) on hat man subjects. Audiology, $12 ;$ t67-173, 1973 ,

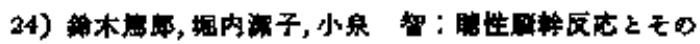

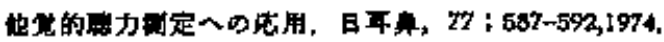

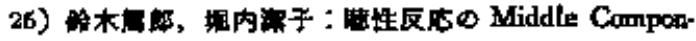
ents ot tof. Audiology Japan, $18 ; 411-412,1976$, 26) Torkildsen, $\boldsymbol{K}$., Osterhamel, $\boldsymbol{P}$ and Huis it, Veld, F.: Electrocochleowaphy with a far field technique. Scand. Audiod, 2 ; 141-148, 1973.

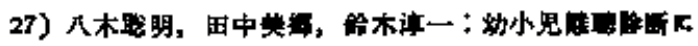
おけ Brain Stem Response の本用性について. Audiology Japan,18 ; 214-220, 1975.

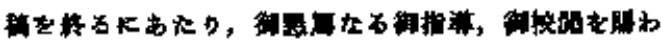

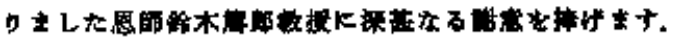

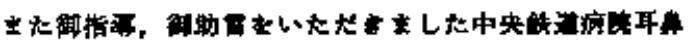

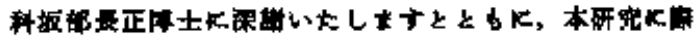

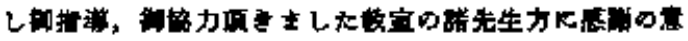
を衰します。

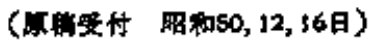

